\title{
ORIGINAL ARTICLE Patient experience survey in telemedicine for spinal cord injury patients
}

\author{
J Yuen, CA Thiyagarajan and M Belci
}

Study design: Patient feedback study.

Objectives: To measure how patients value the telemedicine consultation service at the National Spinal Injuries Centre over a period of 8 months.

Setting: United Kingdom (south of England).

Methods: Telephone survey conducted by an external surveyor who does not work in the department.

Results: Our study showed that most patients $(n=29)$ found the service of high quality and useful to their care.

Conclusion: This provides evidence, from the spinal cord injury patients' perspective, that telemedicine service has an important role in their management.

Spinal Cord (2015) 53, 320-323; doi:10.1038/sc.2014.247; published online 27 January 2015

\section{INTRODUCTION}

As the incidence of spinal cord injury (SCI) remains low ( $\sim 1000$ per year in the $\left.\mathrm{UK}^{1}\right)$ and the patients tend to require highly specialised treatment, there is a tendency to treat SCI patient only in specialised units. However, these units tend to be reasonably spread out and the geographical element makes the logistics to attend face-to-face consultations much more difficult. Moreover, this is exacerbated by the complexity of clinical dynamics (such as pain or spasm), which requires tight follow-up and continued feedback from the patients and community health-care team.

For these reasons, the use of telemedicine is especially useful in SCI patients. Telemedicine may take a variety of forms-for example, telephone calls, video conference calls and remote medical devices. The National Spinal Injuries Centre (NSIC) treats patients from a large geographical area, accepting referrals from the South and East of England, and for children, referrals are also accepted from all of the British Isles and Ireland. ${ }^{2}$ Therefore, telemedicine is a good device for the service intuitively. In this article, we will use the term telemedicine and telehealth interchangeably as a common generic term that covers all aspects of SCI care.

Presently, the literature has covered a number of models and studied its efficacy; however, little has been done on investigating how patients themselves value this kind of service. In a systemic review by Bensink et al., ${ }^{3}$ telehealth intervention appears to provide a superior outcome compared with standard care in a range of specialties. In a study by Phillips et al. ${ }^{4}$ it is shown that telehealth intervention has a significant effect on SCI patients' psychological outcome. A survey conducted by Lewis et al. ${ }^{5}$ shows that both patients and staff agree that telehealth does improve SCI patients' management in pressure sores. In a recent large-scale randomised trial involving 250 patients by Kroenke et al., ${ }^{6}$ it has been shown that telehealth has a significant positive impact on chronic pain patients.

\section{Model in NSIC}

The model of operation, which began in 2013, is shown in Figure 1. Two groups of SCI patients were provided with teleconsultation service. At the start of this service, patients with spasticity intervened by botulinum neurotoxin (BoNT) injection were teleconsulted and later in the programme patients with complex problems were included. Patients with complex problems were seen by SCI consultant (consultant) and patients who required BoNT injection were seen by SCI Associate Specialist who runs the telemedicine service (CT). Any intervention was decided and implemented either by the consultant or

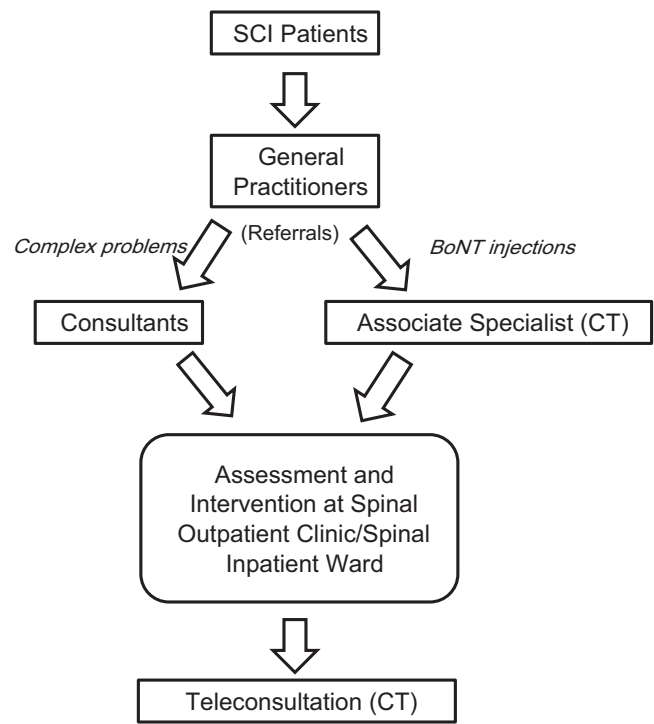

Figure 1 Model of teleconsultation at NSIC. 
by $\mathrm{CT}$ in the spinal unit as an inpatient or outpatient. If change in medication was involved, this was implemented via the local general practitioners (GPs) by giving a clear instruction. Teleconsultation follow-up was then organised according to the intervention and predicted outcomes.

Consultants referred patients to CT via letters or emails and patients who received BoNT injections were routinely booked for teleconsultation at the time of BoNT injection. CT then called up the patients (at home or on their mobile phones) at a specified interval following their inpatient discharge or outpatient appointment. The clinical events were recorded in Microsoft Outlook and then copied and recorded in the hospital electronic patient record. Action required after the teleconsultation was implemented by feeding back to the consultant, who would then instruct the GP or CT on the plan of action to be taken.

Follow-up to interventions such as BoNT injections was generally performed by CT through a multidisciplinary team approach (involving spinal injury consultants, physiotherapists, occupational

Grading
\begin{tabular}{|l|l|}
\hline Quality & Usefulness \\
\hline 1 Very poor & 1 Totally useless \\
\hline 2 Poor & 2 Fairly useless \\
\hline 3 Neutral & 3 Neutral \\
\hline 4 Good & 4 Useful \\
\hline 5 Excellent & 5 Very useful \\
\hline
\end{tabular}

Figure 2 Grading of questions 1 and 2 in the survey. therapists and nursing staff). This often involved the assessment, formulation and implementation of the treatment plans, where the physiotherapist would contact the patient and provide feedback to the community physiotherapist team/GP and spinal consultant. This is done in addition to any formal teleconsultation.

Our study aims to evaluate the quality of our teleconsultation service and patients' satisfaction in order to establish its impact on patient care. No similar studies have been performed in SCI in the UK, to our knowledge.

\section{MATERIALS AND METHODS}

This is a patient feedback study. Duration includes 16 June 2013 to 4 March 2014 inclusive. Telephone survey was conducted between April and May 2014. Telephone surveys were solely conducted by JY, who does not have any direct involvement with the running of the NSIC and therefore there was minimal conflict of interest involved. It was made clear to the patients that the survey results were entirely confidential and would not directly affect the patients' clinical management in any way.

With the recognition that most patients have active lives and may not have time for a full survey, three simple questions were derived.

(1) What was the quality of the way information was given to you?

(2) How useful is the consultation to your care?

(3) Any further feedback—good or bad?

Questions 1 and 2 were graded 1 to 5 , whereas question 3 was an open question. The gradings are as in Figure 2.

\section{RESULTS}

In overall, 104 telephone consultations were included, involving 74 patients.

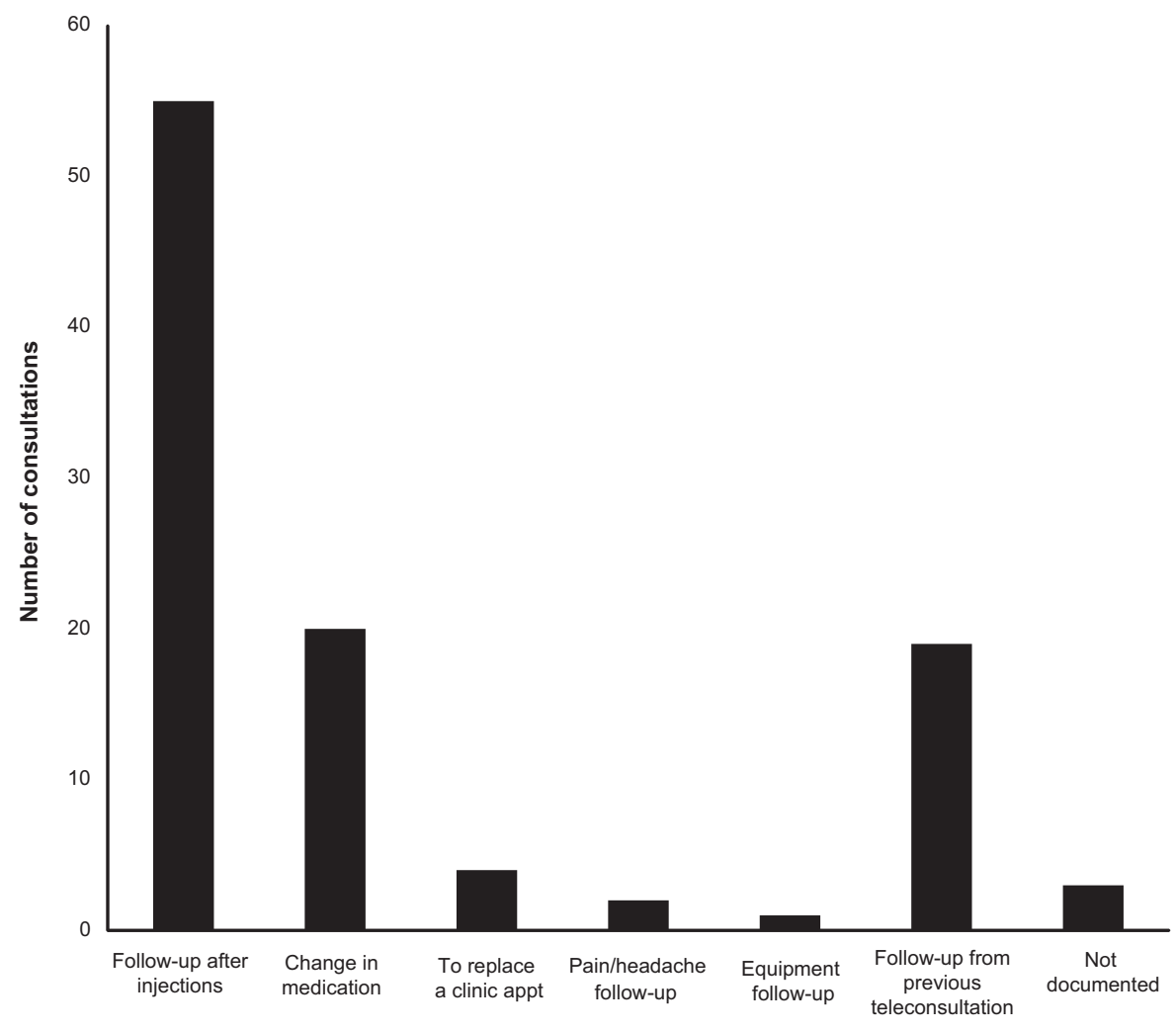

Indication of consultation

Figure 3 Indications of teleconsultation. 


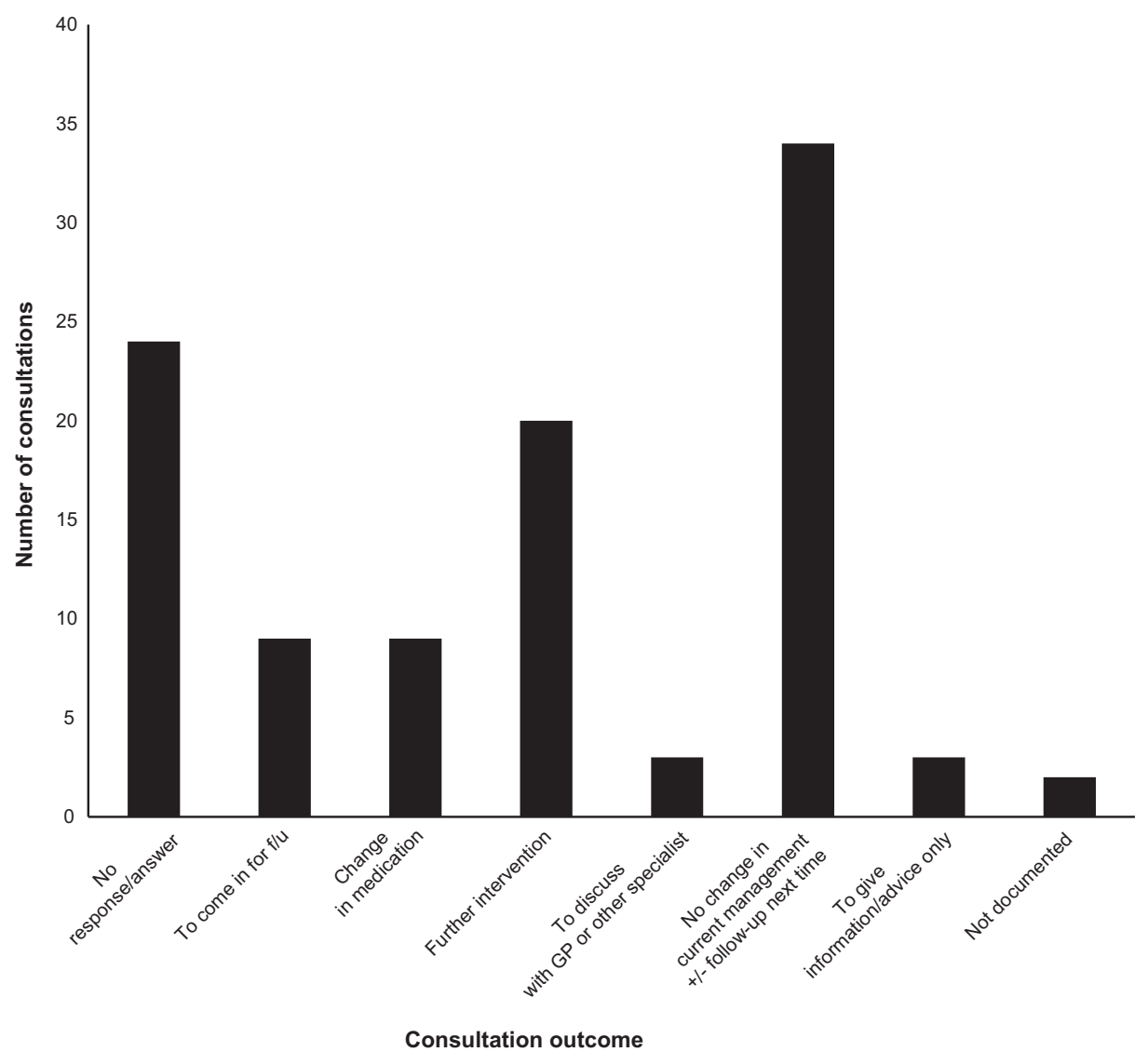

Figure 4 Clinical outcomes of consultation. F/u, follow-up.

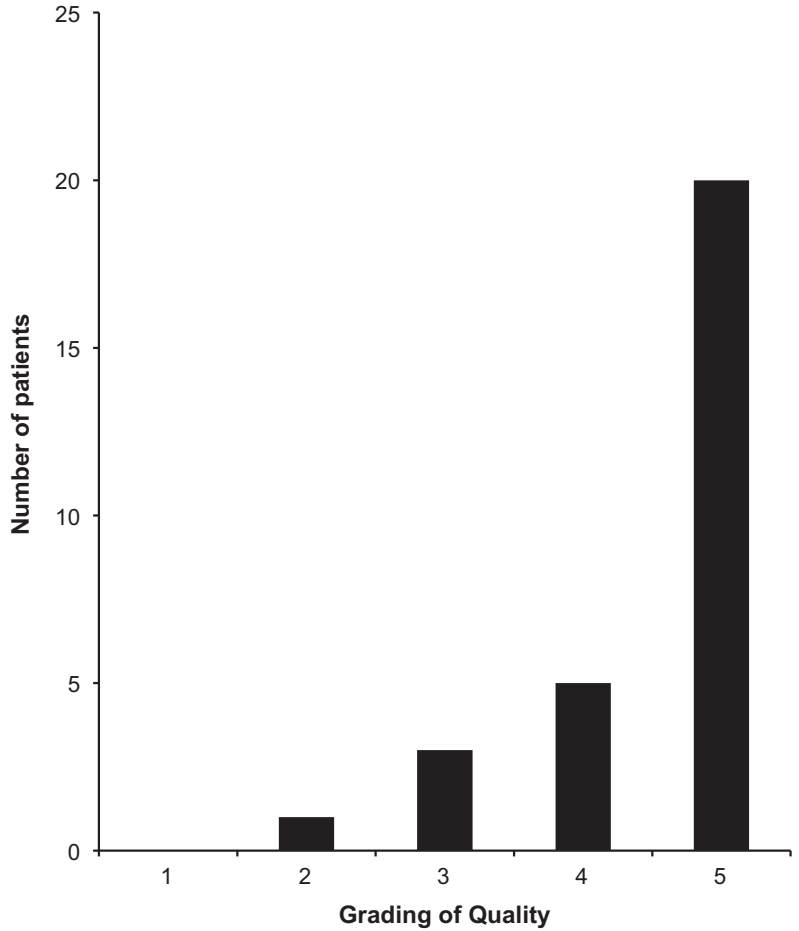

Figure 5 Quality of service rated by patients.
Patients' demographics, indications and outcomes for consultation The mean average age was 50 years (s.e., 2 years) and $66 \%$ were male.

The majority of patients (Figure 3) were followed up after BoNT injections or change in medications (typically in pain or bladder management).

The clinical outcomes of consultation (Figure 4) mainly involve subjective and objective outcome measurements, reassurance and identifying the need for further teleconsultation follow-up.

\section{Survey results}

Despite having 74 patients on the list, only 29 patients were contactable and able to give a response during the survey period.

With regard to the first question, the majority of patients believe the service is of excellent or good quality (Figure 5). This question measures the aggregate of how a doctor gave the information.

With regard to the second question, the majority of patients value the service as very useful or useful to their care (Figure 6).

In terms of positive open feedback, they include

- Very convenient in terms of transport

- Excellent service

- Very personal

- Doctors can see the problem clearly (in a Skype ${ }^{7}$ video conference consultation)

- Attentive listener 


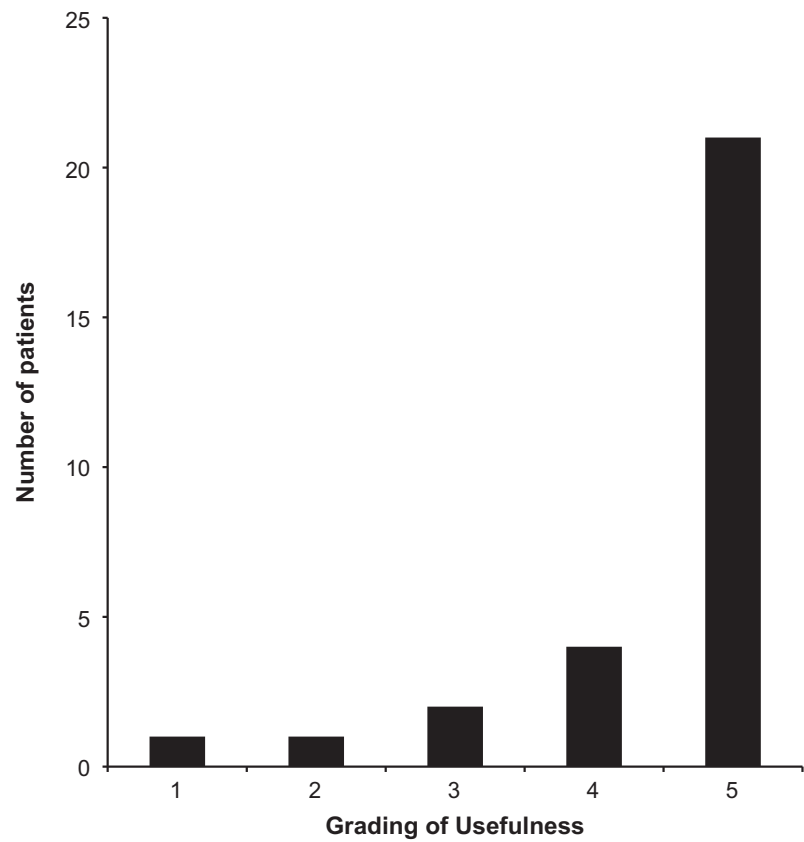

Figure 6 Usefulness of service as rated by patients.

- Very informative

- Friendly

In terms of negative open feedback, they include

- Patient unable to understand long-term plans of their care

- Patient would like more information to be given

- Patient would like a 'drop-in' session on Skype/telephone line

\section{DISCUSSION}

This study reflects the fact that, specific to the telehealth service in NSIC, patients do value it very highly and believe that it would improve their care. However, we acknowledge that the study is limited by the low number of participants and the error in patients recalling their encounter with the clinician retrospectively. The low number of participants in this study reflects the difficulty in performing such service, because doctors and patients often have busy schedules. It is important that both sides should try to adhere to specific consultation times.

\section{CONCLUSION}

Most patients in the NSIC centre currently receive teleconsultation as follow-up to a recent BoNT injection. The majority of patients in the survey found the service of high quality and found the service useful to their care. Previous studies in the literature have shown that telehealth can be of benefit to patients' physical and mental well-being, whereas this study provides strong evidence for continuing such service from the patients' perspective. In addition, we anticipate that the health economists might be interested in developing this assessment and follow-up models.

The use of telehealth technologies has much potential in developing countries, as well as being a major tool of collaboration between centres. International Spinal Cord Injury Society is currently putting a lot of emphasis in developing and extending the treatment of SCI in South Asia, Africa and South America. ${ }^{8}$ Furthermore, according to a recent study within the World Health Organization global regions, ${ }^{9}$ there has been an increase in SCI injuries from motor transport in developing countries due to trends in transport mode (transition to motorised transport), poor infrastructure and regulatory challenges. We believe telemedicine will be an extremely valuable tool on that front due to the limited amount of experts and other resources available.

In our centre, the next steps will be to encourage more members of the team at NSIC to use the service and to conduct a larger study with more rigorous statistical analysis. Also, as video-link technology becomes increasingly widely available, we anticipate more patients would prefer to use such facility, as previously described in the literature. ${ }^{10}$

\section{DATA ARCHIVING}

There were no data to deposit.

\section{CONFLICT OF INTEREST}

The surveys were all conducted by JY, who has no direct involvement in the daily running of NSIC service. There is minimal conflict of interest.

1 Spinal Research, UK. http://www.spinal-research.org/about-us/. Accessed on 1 September 2014.

2 British Association of Spinal Cord Injury Specialists. http://www.bascis.org.uk/? page_id $=152$. Accessed on 1 Septembe 2014.

3 Bensink M, Hailey D, Wootton R. A systematic review of successes and failures in home telehealth: preliminary results. J Telemed Telecare 2006; 12: 8-16.

4 Phillips VL, Vesmarovich S, Hauber R, Wiggers E, Egner A. Telehealth: reaching out to newly injured spinal cord patients. Public Health Rep 2001; 116: 94-102.

5 Lewis M, Choi H, Boushell M, Barsanti P, Alquist M, Sabharwal S. Use of telemedicine in the management of spinal cord injury patients with pressure ulcers. Arch Phys Med Rehabil 2003; 84: E37.

6 Kroenke K, Krebs EE, Wu J, Yu Z, Chumbler NR, Bair MJ. Telecare collaborative management of chronic pain in primary care - a randomized clinical trial. JAMA 2014; 312: 240-248.

7 Skype. http://www.skype.com/en/. Accessed on 1 September 2014

8 International Spinal Cord Society (ISCoS). http://www.iscos.org.uk/about-iscos/ the-objectives-of-iscos. Accessed on 1 September 2014.

9 Lee BB, Cripps RA, Fitzharris M, Wing PC. The global map for traumatic spinal cord injury epidemiology: update 2011, global incidence rate. Spinal Cord 2014; 52 : 110-116.

10 Pain H, Soopramanien A, Dallolio L, Prior R, Menarini M, Ventura M et al. Outcomes from a randomized controlled trial of tele-rehabilitation for people with spinal cord injuries. J Telemed Telecare 2007; 13: 46-48. 